\title{
Artificial Intelligence: Emerging Roles in Medicine
}

\author{
Manmohan M. S. \\ Department of Life science, Bangalore University, \\ Bangalore, Karnataka, India
}

\begin{abstract}
The present study describes the role of artificial intelligence in medical sector. In recent years, there has been large number of applications highlighting the importance of artificial intelligence which are growing rapidly in different sectors. In recent times, there has been implementation of artificial intelligence in medical sector to carryout various desired activity. The present mini review envisions the role of artificial intelligence in medical sector.
\end{abstract}

Keywords: Artificial intelligence, Medical sector, Diagnosis, Telemedicine

\section{INTRODUCTION}

The integration of information sciences has influenced on different sectors [1]. The application and usage of artificial intelligence has traded innumerable applications in recent past [2]. The use of computing (AI) is speedily advancing in several areas of attention and its potential for patient and healthcare skilled service are widely known. the employment of computing in attention not solely provides new treatment and care pathways through nosology and wearable devices (eg FitBit, Apple Watch etc.), however, can even increase the abilities of the health professionals concerned, whereas permitting a larger potency within the implementation of health documentation. In this article, therefore, we'll expose what's the international "practical" story - that's, the concrete application of computing in some hospitals and analysis centres, to higher, perceive the "evolutionary stage" of the world likewise as current technological advances. With a quick mention - in some cases - of the restrictive impact.

\section{HIPPOCRATES 5.0: computing AND medication OF the long run}

In these troublesome and somewhat surreal weeks thanks to the Coronavirus emergency, it's exactly from China that comforting and shocking news arrives within the fight against the virus. within the name of recent technologies and computing, hereunder AI. In January 2020, Chinese leader Xi Jinping turned to the national high-tech giants and invited them to require a number one role within the fight against the epidemic. Alibaba, Baidu and Tencent answered the decision by creating access to the govt of the People's Republic formidable baggage of huge knowledge, computing, artificial intelligence and connected devices, all the simplest that they had [4]. Parenthetically, the Chinese government was able to enforce quarantine for infected patients and map virus movements. Or use intelligent cameras to discover who isn't sporting a mask or to intercept folks with fever because of a period thermal scan. The Covid-19 diagnosing system developed by Alibaba, supported AI, detects new cases of coronavirus with a CT scan with AN accuracy of $96 \%$, in exactly twenty seconds. the simplest ally of the Chinese in times of emergency is proving to be technology. That a revolution is current within the health sector because of AI may be true on that no one dissents any longer. A revolution which will have an effect on our means of treating ourselves, of relating with doctors, of being operated on, of tracing our medical account and even of coaching the doctors of tomorrow. Surgical robots, machine learning for diagnoses, 3D written organs and alternative last technologies are going to be as acquainted with the USA as of today's Electronic Health Record. however, let's enter an order.

\section{TELEMEDICINE, DIAGNOSING AND PROPHETICAL MODELS}

Both the business system and also the attention system agree on the importance of finance in artificial intelligence-enabled healthcare technologies. The political objectives are quite evident, from up the standard of help to simplifying the decision-making method to increasing the potency and aggressiveness of the socio-health system. On a medical level, there are several prospects beginning with tele medicine, that we've got already familiar in recent years, or remote observation of the health conditions of patients. because of AI and medical aid, attention professionals are going to be able to manage patients with chronic diseases additional quickly and economically, who are going to be monitored remotely, liberating up substantial attention prices. At a similar time, it'll be potential to extract info from electronic medical records and additional economically manage the executive practices that nowadays absorb $30 \%$ of health prices, therefore on relieve doctors from routine tasks and provides them longer to devote to the foremost vital patients. especially, because of increased and video game, it's additionally potential to contribute to the repair and maintenance of electromedical instrumentation remotely, with 5.0 technical help systems for workers gift on the web site. "Precision medicine" additionally finds a valuable ally in AI. because of psychological feature supercomputers capable of analyzing giant amounts of knowledge, it's potential to form early and additional precise diagnoses and to organize life-saving therapies quicker than ancient ways. computing which will additionally support surgical microrobotics, or rather robot-assisted surgery. AN example comes from the heart of the University of Maastricht, 
within the Holland, wherever researchers have experimented with the employment of AN AI-assisted golem to microscopically suture small blood vessels within the order of 0.03 millimetres. though to possess fully autonomous surgeries with solely the surgeon's management, you may have to be compelled to wait till 2050. A similar discussion is opened for the treatment and bar of rare diseases, genetic diseases or diseases from wrong lifestyles. the power to extract and method giant volumes of knowledge, medical pictures, medicine statistics, from that to make prophetical models, represents a vast potential each to support doctors and to stop the unfold of diseases. In these Coronavirus weeks, if we tend to had had additional refined analysis tools accessible, the Authorities may have acted in an exceedingly additional precise and expedited means [5-7].

\section{ARTIFICIAL INTELLIGENCE AND KNOWLEDGE GOVERNMENT}

According to 2018 HIMMS survey, solely $16 \%$ of attention facilities in Europe use AI systems in health care, though an oversized proportion will do therefore within the future. To date, European attention professionals use "intelligent" systems in progress management (14\%) in the analysis $(13 \%)$, within the administration of medicines $(12 \%)$ and in radiology (11\%). The medicine space is that the one wherever you'll comprehend the biggest investments are going to be created. The pharmaceutical business is additionally experiencing a robust innovative push supported AI systems. the foremost vital corporations within the sector have signed partnerships with innovative companies and start-ups to bring the advantages of machine learning and deep learning to analyze, especially to develop pharmaceutical merchandise with quicker times and prices than ancient and valuable clinical studies. Hence computing may be a candidate to be the new digital frontier within the attention market: recent studies say that the expansion rate of the employment of computing can go from a pair of.1 billion bucks in 2018 to36.1 billion bucks of 2025. For a big market that unveil, there are even as several challenges that open up. beginning with the govt of AN ever-increasing quantity of knowledge. Forecasts indicate that by the top of 2020 medical data can double every seventy-three days and medical employees are going to be enough to investigate this flow of knowledge. parenthetically, CT (Computerized Tomography) and tomography (Magnetic Resonance Imaging) examinations can increase with a well equal range of radiologists. thanks to this gap, the support of computing are going to be decisive to permit a speedy analysis of the exams and a discount within the error rate, an occasion that's invariably gift and that human intervention remains irreplaceable or essential. Governing knowledge so, however, additionally coming up with technological infrastructures capable of separating with none chance of error, as an instance, the planet of the file wherever sensitive knowledge is obvious and used for clinical functions, from the planet of huge knowledge, wherever knowledge is processed anonymously and aggregately and used for analysis functions solely (as needed by all regulations). Risk management is, of course, the issue that also slows down the adoption of AI because it needs the flexibility of general trust between many subjects and therefore the confidence of doctors and nurses. The management of legal aspects, privacy and ability between systems are involved. it's no coincidence that, so as to make adequate trust in an exceedingly sensitive and sensitive market comparable to health, the ECU Commission appointed in June 2018 a panel of "high-level" consultants capable of examining new moral rules and to counter potential "dangerous" drifts of AI. Today carcinoma is that the second leading explanation for cancer death in ladies, so early diagnosing is decisive for the treatment and hindrance of carcinoma. On this, Nature reveals Google is ending some tests with AI to boost the first detection method of carcinoma, i.e. scale back false negatives by nine. $7 \%$ and false positives by $5.7 \%$ compared to ancient medical reports. it's clear that the potential is gigantic which, with machine learning techniques, the system adjusts the analysis of cases wherever it's wrong and might improve speedily. unitedly with the University of the American state, Stanford and therefore the University of Chicago, Google has developed prognostic AI solutions that, supported the results of hospital visits, will forestall readmissions and scale back time of patients' keep within the same hospitals. If Google experiments, IBM is already one step ahead. IBM Watson is, of course, one in every of the reference software package within the world of AI and is employed to spot specialised treatments for cancer patients. This software package performed huge knowledge analysis on over $\mathrm{k}$ individuals at the University of North Carolina's Comprehensive Cancer Center to work out the proper treatment choices for patients with tumours having genetic abnormalities [8-10].

\section{ADVANTAGES AND RISKS OF AI IN HEALTH}

So what are the benefits however additionally the risks for AI applications in healthcare? In terms of prices, $30 \%$ of the care value is thanks to body activities. As simply understood, AI will alter several activities, from the management of waiting lists to the dialogue with insurance firms, from the follow-up of unpaid invoices to knowledge entry, therefore releasing up several resources. The success of AI-based care technology also will rely on the web of Things, especially those "wearable" sensors capable of grouping knowledge, or of accelerating the standard of lifetime of users, or of alerting users and their care professionals to potential health risks. IoT which can so work each at a prognostic and knowledge assortment level and as a warning to users. All with nice savings in time, uncalled-for visits or hospital remissions, and money, the general public and patients [11-13].

\section{THE HEALTH OF TOMORROW IS GOING TO BE VERY PREVENTABLE}

Tomorrow's care is going to be very preventive and prognostic, it'll be additional targeted and personalised to the extent that we've got personalized treatment plans or protocols. because of AI, we are going to enter a brand new era of ultra-early nosology, especially for the arena of 
diagnostic imaging which can see particularly important blessings. it'll be easier to estimate the value of hospitalization for crucial sicknesses, analyze the aspect effects of treatments, monitor the impact of potential hindrance campaigns or anticipate emergency things or predict the unfold of a malady. If machine learning is progressively refined and higher, this can enable individuals to visualize, correct and improve these dynamics and therefore build the algorithmic rule additional economical and "refined". A machine these days is unable to supply diagnoses, it can not be left with a decision-making method that's still firmly within the hands of man. The support provided by the AI ought to, the conditional may be a should have the flexibility to spot areas of uncertainty, shadow areas to be subjected to the assessment of human intelligence. This passage would produce the required trust between men and machines, a necessity for happy cooperation. Last, under no circumstances least, the info Security issue within the care sector on the idea of EU Regulation 679/16 (GDPR). The approach supported the ideas of answerability and risk management is pushing medical and clinical solutions towards company management systems based on privacy by default and advisedly. firms are known as upon to produce adequate security measures in terms of preventive and prognostic maintenance, cybersecurity, business continuity and disaster recovery. For years, our company has been committed to providing specialised recommendation to its customers, within the awareness that the unquestioned blessings of victimisation $\mathrm{AI}$ in technology and therefore the storage of patient knowledge is often invalidated in the absence of adequate and rigorous safety and protection procedures [14-20].

\section{CONCLUSION}

Medicine 5.0is the corresponding event in medicin e of Fourth Industrial Revolution, characterized by a fusion of physical, digital and biological worlds, impacting all dis ciplines, economies and industries, and even challenging id eas about what it means to be human. Three are the main tr ends in the near future of medicine: Integrative Medicine, Regenerative Medicine, and Precision Medicine. The infor matics revolution and artificial intelligence (AI) are crucial in the development of the three perspectives. The accelerati on of Medicine 5.0determines big changing in medical rese arch, in a healthcare organization, and in the idea itself of medicine. AI challenges to medicine and physicians from $\mathrm{p}$ ractical and theoretical points of view, with revolutionary ways for medical assistance, diagnosis, therapeutics, and th e physicianpatient relationship. Physicians and medical pro fessionals have the duty of information and education of pa tients and public about the future changing, for helping und erstanding and aware democratic decision.

\section{REFERENCE}

[1] Rahul Reddy Nadikattu, New Ways in Artificial Intelligence international journal of computer trends and technology, 2019. Available at SSRN: https://ssrn.com/abstract $=3629063$ or http://dx.doi.org/10.2139/ssrn.3629063(November 7, 2019).

[2] Mohammad and Sikender Mohsienuddin, Artificial Intelligence in Information Technology Available at SSRN: https://ssrn.com/abstract=3625444

http://dx.doi.org/10.2139/ssrn.3625444(June 11, 2020).

[3] Soni, Vishal Dineshkumar, Challenges and Solution for Artificial Intelligence in Cybersecurity of the USA Available at SSRN: https://ssrn.com/abstract=3624487

http://dx.doi.org/10.2139/ssrn.3624487(June 10, 2020).

[4] Nadikattu, Rahul Reddy, Information Technologies: Rebooting the World Activities during COVID-19 (June 9, 2020). Available at SSRN: https://ssrn.com/abstract $=3622733$ or http://dx.doi.org/10.2139/ssrn.3622733

[5] D. Kanabolo and M.S. Gundeti, The Role of Artificial Intelligence (AI) in Medical Imaging: General Radiologic and Urologic Applications. Medical Imaging, 26-42. doi:10.1201/9780429029417-2(2019).

[6] S.Bredt, Artificial Intelligence (AI) in the Financial SectorPotential and Public Strategies. Frontiers in Artificial Intelligence, 2. doi:10.3389/frai.2019.00016(2019).

[7] 3 Artificial Intelligence in the Aviation Sector. (2019). Research Focus. doi:10.36068/1.7

[8] Graph 1.24 Artificial intelligence patents by top 2000 R\&D companies, by sector, 2012-14. (n.d.). doi:10.1787/888933617301

[9] M. Gilbert, The Role of Artificial Intelligence for Network Automation and Security. Artificial Intelligence for Autonomous Networks, 1-23. doi:10.1201/9781351130165-1

[10] W. Horn, Artificial intelligence in medicine and medical decision making Europe. Artificial Intelligence in Medicine, 20(1), 1-3. doi:10.1016/s0933-3657(00)00049-x(2000).

[11] Artificial Intelligence in Medical Science. medical diagnosis using artificial neural networks, 11-23. doi:10.4018/978-1-46666146-2.ch002(2014)

[12] Mohammad, Sikender Mohsienuddin, Security and Privacy Concerns of the 'Internet of Things' (IoT) in IT and its Help in the Various Sectors across the World (April 4, 2020). International Journal of Computer Trends and Technology (IJCTT) - Volume 68 Issue 4 - April 2020. Available at SSRN: https://ssrn.com/abstract=3630513

[13] B.Allen, R.Gish and K. Rreyer, The Role of an Artificial Intelligence Ecosystem in Radiology. Artificial Intelligence in Medical Imaging, 291-327. doi:10.1007/978-3-319-948782_19(2019).

[14] Z. Hoodbhoy, B.Hasan and K.Siddiqui, Does artificial intelligence have any role in healthcare in low resource settings? Journal of Medical Artificial Intelligence, 2, 13-13. doi:10.21037/jmai.2019.06.01(2019).

[15] A.Sanches-Medeiros, L.Martins-Santana and R.Silva-Rocha, Setting patterns and predicting: The role of artificial intelligence in synthetic and natural promoter screening. journal of medical $\begin{array}{lll}\text { artificial intelligence, } & 25-25\end{array}$ doi:10.21037/jmai.2019.11.01(2019).

[16] A. Sloman, Interactions between philosophy and artificial intelligence: The role of intuition and non-logical reasoning in intelligence. Artificial Intelligence, 2(3-4), 209-225. doi:10.1016/0004-3702(71)90011-7(1971).

[17] J. Brag, Artificial Intelligence in Medical Imaging. healthcare and artificial intelligence, 93-103. doi:10.1007/978-3-030-321611_14(2020).

[18] Ayache, N. (2020). Medical Imaging in the Age of Artificial Intelligence. Healthcare and Artificial Intelligence, 89-91. doi:10.1007/978-3-030-32161-1_13

[19] Ling, N. (2018). Dr. Anthony C. Chang: Artificial intelligence (AI) depicts future medical blueprints. Journal of Medical Artificial Intelligence, 1, 1-1. doi:10.21037/jmai.2018.06.01

[20] Agah, A. (2013). Introduction to Medical Applications of Artificial Intelligence. Medical Applications of Artificial Intelligence, 1-8. doi:10.1201/b15618-2 